\title{
Hypoactivity following perturbed estrogen signaling in the medial amygdala
}

\author{
Chia Li and Michael J. Krashes \\ Diabetes, Endocrinology and Obesity Branch, National Institute of Diabetes and Digestive and Kidney Diseases, NIH, Bethesda, Maryland, USA. National Institute on Drug Abuse, NIH, Baltimore, Maryland, USA.
}

\begin{abstract}
Activation of estrogen receptor $\alpha(E R \alpha)$ in the brain prevents obesity as the result of increased energy expenditure and decreased food intake. While ERa is present on several neural populations, it is not clear how different regions of the brain mediate the weight-regulating effects of ER $\alpha$ activation. In this issue of the $J C l, X u$ and colleagues provide extensive evidence that $E R \alpha$ is abundant on neurons expressing single-minded-1 (SIM1) in the medial amygdala (MeA) and that loss of ER $\alpha$ in these cells enhances weight gain in both male and female mice, as the result of reduced physical activity. Moreover, focal deletion of ER $\alpha$ from the $\mathrm{MeA}$ recapitulated these alterations in energy homeostasis. Conversely, overexpression of ER $\alpha$ in the MeA partially prevented mice from dietinduced obesity, while chemogenetic activation of SIM1-expressing neurons in the MeA transiently promoted physical activity. The results of this study provide important insight into the regions of the brain that mediate ER $\alpha$ dependent energy homeostasis.
\end{abstract}

and food intake, the ER $\alpha$-expressing neurons that alter physical activity have yet to be elucidated. In this issue, $\mathrm{Xu}$ et al. demonstrate that targeted deletion of Ers1 from single-minded-1 (SIM1) neurons, which are abundantly expressed in the medial amygdala (MeA), causes hypoactivity and obesity in both male and female mice (11).

\section{Metabolic consequences of ER $\alpha$ deficiency in SIM1 neurons} $\mathrm{Xu}$ et al. explored potential areas of the brain that coexpress ER $\alpha$ and the transcription factor SIM1 by systematically quantifying overlap, and they deduced that the most abundant SIM1-expressing ER $\alpha$ neurons are located in the $\mathrm{MeA}(\sim 80 \%)$ in both males and females (11). Interestingly, the number of SIM1-expressing ER $\alpha$ neurons in the MeA was higher in males $(7,000)$ compared with females $(4,000)$, a discrepancy that may account for disparate phenotypes between sexes. Although ER $\alpha$ expressing SIM1 neurons were found in the paraventricular hypothalamic nucleus (PVN) and POAH, colocalization of SIM1 and $\mathrm{ER} \alpha$ was minimal in these regions, compared with the MeA.

Having located the anatomical sites of intersectional SIM1 and ER $\alpha$ expression, $\mathrm{Xu}$ and colleagues selectively deleted Esr1 from SIM1-expressing neurons (Sim1-Cre Esr $1^{f / f l}$, referred to as SIM1-ER $\alpha$-KO mice), which resulted in a multitude of metabolic consequences (11). When fed a standard chow diet, both female and male SIM1ER $\alpha-\mathrm{KO}$ mice exhibited late-onset obesity that was associated with increased fat mass as a result of reduced energy expenditure. Specifically, this fall in energy expenditure was ascribed to a robust decline in physical activity (assessed by ambulatory movements and rearing) that primarily occurred during the dark cycle: the subjective active period of these animals. Notably, the decreases in energy expenditure were subtle, producing weight gain over an appreciable time, which suggests that ER $\alpha$ signaling in SIM1 neurons may prevent 
age-associated obesity. Alternatively, as these animals are ER $\alpha$ deficient from the embryonic stage, the phenotypic effects may be tempered due to developmental compensation.

Nevertheless, SIM1-ER $\alpha$-KO males were susceptible to diet-induced obesity, as these animals gained substantially more weight than controls when placed on a high-fat diet (HFD). Intriguingly, SIM1ER $\alpha-K O$ females did not have the same predisposition for diet-induced obesity, as they had comparable weights to control animals. The drastic sex-dependent difference in overall number of ER $\alpha$-expressing SIM1 neurons in the MeA may explain this phenotypic dimorphism. Alternatively, $\mathrm{Xu}$ et al. implicate that the female brain may comprise more resilient redundant pathways than their male counterparts. Reinforcing this view, multiple $\mathrm{ER} \alpha$-expressing sites in the brain, including the ARC and $\mathrm{VMH}$, have been linked to energy-balance regulation in females (7); however, this ER $\alpha$-expressing SIM1 population in the $\mathrm{MeA}$ is the lone population identified to this point that mediates body weight homeostasis in males.

A potential caveat of the results obtained from SIM1-ER $\alpha-\mathrm{KO}$ mice is that the observed effects may be due to alteration of ER $\alpha$-meditated genes that are also involved in cell signaling and neural communication (12). To address this issue, $\mathrm{Xu}$ and colleagues analyzed gene-expression profiles in the amygdala and found no marked changes in mRNA encoding the androgen receptor (AR), aromatase, glutamate decarboxylase 1 (GAD1), glutamate decarboxylase 2 (GAD2), glutaminase (GLS), glutamate-ammonia ligase (GLUL), melanocortin 4 receptor (MC4R), or nitric oxide synthase-1 (NOS1) in male or female SIM1-ER $\alpha-\mathrm{KO}$ mice compared with controls (11). Furthermore, expression of hypothalamic genes associated with body weight regulation - such as those encoding agoutirelated peptide (AgRP), leptin receptor, NOS1, neuropeptide Y (NPY), and POMC - were similar to controls. However, the possibility remains that undetected alternate gene-expression patterns and/or levels may affect energy balance in SIM1$\mathrm{ER} \alpha-\mathrm{KO}$ mice. It should also be noted that deletion of Esr1 from SIM1 neurons may be deleterious to endocrine function and/or chronic behavior; however, $\mathrm{Xu}$ et al. did not report any deficits in fertility between control and SIM1-ER $\alpha$-KO dams. Moreover, Xu et al. employed a battery of tests and demonstrated that the hypoactivity observed in SIM1-ER $\alpha-\mathrm{KO}$ mice is independent of anxiety, a state repeatedly assigned to neural circuits in the amygdala (13). Specifically, there was no difference in responses between SIM1-ER $\alpha$-KO mice and control animals when tested in open field, light/dark, and elevated plus maze assays. Thus, the sharp decrease in locomotor activity observed in SIM1-ER $\alpha-\mathrm{KO}$ animals is independent of a permanent anxiogenic condition.

\section{The MeA mediates ER $\alpha$ - dependent effects on physical activity}

The abundant compensatory mechanisms that are involved in the neural control of energy balance are a major limitation to genetic studies that rely on the developmental deletion of a particular allele (14-17). Furthermore, the gene of interest will be deleted in all cells that express the gene driving Cre expression. As SIM1expressing neurons are present in multiple regions of the brain, the conclusions of $\mathrm{Xu}$ et al. based on SIM1-ER $\alpha-\mathrm{KO}$ mice are limited in regard to the precise anatomical region of action. Xu et al. employed a viral-mediated delivery approach to further specify the region of the brain that mediates ER $\alpha$-dependent effects on activity (11). The MeA of Esr ${ }^{f l / f l}$ mice was stereotaxically targeted with an adeno-associated virus expressing Cre-recombinase (AAV-Cre). Compared with control mice, selective removal of $\mathrm{ER} \alpha$ from the $\mathrm{MeA}$ in adult animals resulted in a rapid, elevated weight gain on a standard chow diet and a dramatic body weight increase in response to an HFD due to considerable reductions in physical activity. In contrast, deletion of Esr1 exclusively from the PVH did not affect physical activity, further supporting the role of MeA-specific ER $\alpha$ activity in the regulation of energy balance.

Together, these loss-of-function studies establish that ER $\alpha$ function in the MeA is required for energy homeostasis, but is $\mathrm{ER} \alpha$ activation sufficient to maintain the balance in response to HFD? Xu et al., addressed this question by selectively overexpressing ER $\alpha$ in the MeA in mice harbor- ing a Cre-activated human ERS1 allele in the Rosa26 locus (11). Following restricted AAV-Cre injection, overexpression of human ER $\alpha$ in the MeA partially protected mice from diet-induced obesity, compared with controls fed an HFD. While these results suggest that $\mathrm{ER} \alpha$ induction in the MeA can limit diet-induced obesity, this approach will result in ER $\alpha$ expression at quantities that far exceed endogenous levels, as well as ectopic expression. A more physiologically relevant tactic would be to apply a Cre-dependent reactivation strategy that has been previously used to test sufficiency in models of energy balance $(18,19)$. Such an approach would result in endogenous reexpression of ER $\alpha$ only at sites that normally express this receptor.

$\mathrm{Xu}$ and colleagues also investigated the effects of the highly selective ER $\alpha$ agonist propyl pyrazole triol (PPT) on neural activity (11). In a SIM1 reporter mouse, PPT depolarized, increased the firing rate of, and decreased input resistance in the majority of SIM1 neurons in the MeA, while blocking action potential-dependent network activity. These effects were most likely mediated through an ER $\alpha$ dependent mechanism, as few SIM1expressing MeA neurons were responsive to PPT in SIM1-ER $\alpha-K O$ mice. Moreover, chemogenetic activation of SIM1-expressing MeA neurons with designer receptors exclusively activated by designer drugs technology (DREADD technology) (20, 21) resulted in acute stimulation that transiently heightened physical activity. Interestingly, the hM3Dq DREADD system that was employed has been demonstrated to signal for hours $(14,15,20,21)$; however, the behavioral effects in this model were short-lived. $\mathrm{Xu}$ et al. hypothesized that although SIM1-expressing MeA neurons can alter activity, they fail to regulate arousal and circadian control; therefore, activation during the light cycle led to transitory changes. It would be of value to repeat these experiments during the active period of these animals to further dissect the precise function of these neurons.

\section{Conclusions and future directions}

In humans, plasma levels of glucagon-like peptide-1 (GLP-1) strongly correlate with body fat mass in healthy adults (22). Treatment with GLP-1 receptor agonists leads 
to weight loss in obese patients, particularly in those with diabetes (23). Furthermore, mice treated with a GLP-1-estrogen conjugate exhibit a greater weight reduction than those treated with GLP-1 alone, an approach that takes advantage of the weight loss effect of estrogen but avoids its carcinogenic qualities (24). Interestingly, GLP-1 receptors are densely expressed in the MeA (25). $\mathrm{Xu}$ and colleagues further identified a node through which GLP-1estrogen exerts its therapeutic effects to combat obesity. Subcutaneous administration of GLP-1-estrogen increased expression of the estrogen target Trim 25 in both the amygdala and the hypothalamus, suggesting that $\mathrm{ER} \alpha$ in the MeA contributes to the weight-regulating effects of this co-agonist conjugate. In support of this possibility, treatment of SIM1-ER $\alpha-\mathrm{KO}$ mice with the GLP-1-estrogen conjugate was less effective at reducing body weight. Together, these results indicate that ER $\alpha$ expressing SIM1 neurons in the MeA neurons may be critical for the weight lossinducing properties of GLP-1-estrogen.

The work by Xu et al. provides further insight into the neuroanatomy involved in ER $\alpha$-mediated obesity. To begin to address the neural circuitry through which ER $\alpha$-expressing MeA neurons regulate changes in physical activity, this study demonstrates that the preponderance of SIM1-expressing MeA neurons are glutamatergic and these neurons send dense efferents to the red nucleus, as well as the dorsal and median raphe nuclei. Modern neuroscience tools have the ability to acutely and reversibly drive neural activity in animal models, allowing a more complete understanding of neuronal circuits involved in regulating specific phenotypes. Future studies should utilize these tools to trace inputs and outputs to ER $\alpha$-expressing MeA neurons and elucidate unanswered inquiries, such as monosynaptic connectivity to and function of downstream regions on behavior (26). Moreover, these applications have potential to pinpoint the anatomical configuration of the circuitry involved in regulating phenotypes of interest, leading to putative therapeutic strategies.

\section{Acknowledgments}

This research was supported by the Intramural Research Program of the NIH, NIDDK.

Address correspondence to: Michael J. Krashes, 10 Center Drive, Bldg. 10, Rm. 6-3932, Bethesda, Maryland 20892, USA. Phone: 301.827.0960; E-mail: michael. krashes@nih.gov.

1. Ahdieh HB, Wade GN. Effects of hysterectomy on sexual receptivity, food intake, running wheel activity, and hypothalamic estrogen and progestin receptors in rats. J Comp Physiol Psychol. 1982;96(6):886-892.

2. Butera PC, Czaja JA. Intracranial estradiol in ovariectomized guinea pigs: effects on ingestive behaviors and body weight. Brain Res. 1984;322(1):41-48.

3. Colvin GB, Sawyer CH. Induction of running activity by intracerebral implants of estrogen in overiectomized rats. Neuroendocrinology. 1969;4(4):309-320.

4. Gao Q, et al. Anorectic estrogen mimics leptin's effect on the rewiring of melanocortin cells and Stat 3 signaling in obese animals. Nat Med. 2007;13(1):89-94.

5. Heine PA, Taylor JA, Iwamoto GA, Lubahn $\mathrm{DB}$, Cooke PS. Increased adipose tissue in male and female estrogen receptor-alpha knockout mice. Proc Natl Acad Sci U S A. 2000;97(23):12729-12734.

6. Rogers NH, Perfield JW 2nd, Strissel KJ, Obin MS, Greenberg AS. Reduced energy expenditure and increased inflammation are early events in the development of ovariectomy-induced obesity. Endocrinology. 2009;150(5):2161-2168.

7. Xu Y, et al. Distinct hypothalamic neurons mediate estrogenic effects on energy homeostasis and reproduction. Cell Metab. 2011;14(4):453-465.

8. Santollo J, Torregrossa AM, Eckel LA. Estradiol acts in the medial preoptic area, arcuate nucleus, and dorsal raphe nucleus to reduce food intake in ovariectomized rats. Horm Behav. 2011;60(1):86-93.

9. Cao X, et al. et al. Estrogens stimulate serotonin neurons to inhibit binge-like eating in mice. J Clin Invest. 2014;124(10):4351-4362.

10. Asarian L, Geary N. Estradiol enhances cholecystokinin-dependent lipid-induced satiation and activates estrogen receptoralpha-expressing cells in the nucleus tractus solitarius of ovariectomized rats. Endocrinology. 2007;148(12):5656-5666.

11. Pingwen X, et al. Estrogen receptor- $\alpha$ in medial amygdala neurons regulates body weight. J Clin Invest. 2015;215(7):2861-2876.

12. Malyala A, Pattee P, Nagalla SR, Kelly MJ, Ronnekleiv OK. Suppression subtractive hybridization and microarray identification of estrogenregulated hypothalamic genes. Neurochem Res. 2004;29(6):1189-1200.

13. Janak PH, Tye KM. From circuits to behaviour in the amygdala. Nature. 2015;517(7534):284-292.

14. Atasoy D, Betley JN, Su HH, Sternson SM. Deconstruction of a neural circuit for hunger. Nature. 2012;488(7410):172-177.

15. Krashes MJ, Shah BP, Koda S, Lowell BB. Rapid versus delayed stimulation of feeding by the endogenously released AgRP neuron mediators GABA, NPY, and AgRP. Cell Metab. 2013;18(4):588-595.

16. Qian S, et al. Neither agouti-related protein nor neuropeptide $\mathrm{Y}$ is critically required for the regulation of energy homeostasis in mice. Mol Cell Biol. 2002;22(14):5027-5035.

17. Tong Q, Ye CP, Jones JE, Elmquist JK, Lowell BB. Synaptic release of GABA by AgRP neurons is required for normal regulation of energy balance. Nat Neurosci. 2008;11(9):998-1000.

18. Balthasar N, et al. Divergence of melanocortin pathways in the control of food intake and energy expenditure. Cell. 2005;123(3):493-505.

19. Xu Y, et al. A serotonin and melanocortin circuit mediates D-fenfluramine anorexia. J Neurosci. 2010;30(44):14630-14634.

20. Alexander GM, et al. Remote control of neuronal activity in transgenic mice expressing evolved G protein-coupled receptors. Neuron. 2009;63(1):27-39.

21. Krashes MJ, et al. Rapid, reversible activation of AgRP neurons drives feeding behavior in mice. J Clin Invest. 2011;121(4):1424-1428.

22. van der Stouwe JG, et al. Plasma levels of glucagon-like peptide 1 markers of obesity among young healthy adults [published online ahead of print April 10, 2015]. Clin Endocrinol (Oxf). doi:10.1111/cen.12789.

23. Heppner KM, Perez-Tilve D. GLP-1 based therapeutics: simultaneously combating T2DM and obesity. Front Neurosci. 2015;9:92.

24. Finan B, et al. Targeted estrogen delivery reverses the metabolic syndrome. Nat Med. 2012;18(12):1847-1856.

25. Gu G, Roland B, Tomaselli K, Dolman CS, Lowe C, Heilig JS. Glucagon-like peptide-1 in the rat brain: distribution of expression and functional implication. JComp Neurol. 2013;521(10):2235-2261.

26. Garfield AS, et al. A neural basis for melanocortin-4 receptor-regulated appetite [published online ahead of print April 27, 2015]. Nat Neurosci. doi:10.1038/nn.4011. 